\title{
Obstacle Numbers of Graphs
}

\author{
Hannah Alpert • Christina Koch • Joshua D. Laison
}

Received: 16 January 2009 / Revised: 13 November 2009 / Accepted: 15 November 2009 /

Published online: 3 December 2009

(C) Springer Science+Business Media, LLC 2009

\begin{abstract}
An obstacle representation of a graph $G$ is a drawing of $G$ in the plane with straight-line edges, together with a set of polygons (respectively, convex polygons) called obstacles, such that an edge exists in $G$ if and only if it does not intersect an obstacle. The obstacle number (convex obstacle number) of $G$ is the smallest number of obstacles (convex obstacles) in any obstacle representation of $G$. In this paper, we identify families of graphs with obstacle number 1 and construct graphs with arbitrarily large obstacle number (convex obstacle number). We prove that a graph has an obstacle representation with a single convex $k$-gon if and only if it is a circular arc graph with clique covering number at most $k$ in which no two arcs cover the host circle. We also prove independently that a graph has an obstacle representation with a single segment obstacle if and only if it is the complement of an interval bigraph.
\end{abstract}

Keywords Obstacle number · Convex obstacle number · Circular arc graph · Proper circular arc graph · Non-double-covering circular arc graph · Interval bigraph · Visibility graph

\footnotetext{
H. Alpert

Department of Mathematics, University of Chicago, 5734 S. University Avenue, Chicago, IL 60637, USA

e-mail: hcalpert@uchicago.edu

C. Koch

Academy of Hope, 601 Edgewood St. NE, Suite 25, Washington, DC 20017, USA

e-mail: christina@aohdc.org

J.D. Laison ( $\varangle)$

Mathematics Department, Willamette University, 900 State St., Salem, OR 97301, USA

e-mail: jlaison@willamette.edu
} 
Fig. 1 Every finite graph has finite obstacle number

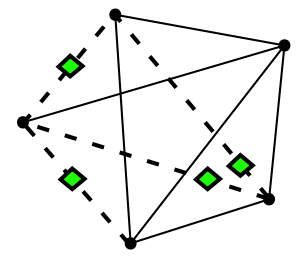

\section{Introduction}

Given a set $V$ of points in the plane and a set $O$ of polygons, called obstacles, in the plane, we form a graph $G$ with a vertex for each point in $V$, and an edge between two vertices if and only if the line segment between them does not intersect an obstacle. We call the pair $(V, O)$ an obstacle representation of $G$. We assume that the points in $V$ and the vertices of polygons in $O$, taken together, are in general position, i.e., no three are collinear.

A (straight-edge) drawing of a graph $G$ assigns the vertices of $G$ to points in general position in the plane, and the edges of $G$ to line segments between the corresponding points. We use the same names for the vertices and edges of $G$ and of the drawing of $G$. All our representations of graphs in the plane will be straight-edge drawings.

Note that every finite graph $G$ has an obstacle representation with a finite number of obstacles, constructed in the following way. In any drawing of $G$, for each nonedge of $G$, construct a small obstacle intersecting that non-edge and no edge of $G$, as shown in Fig. 1. For a given graph $G$, we call the least number of obstacles in any obstacle representation of $G$ the obstacle number of $G$.

In fact, the above construction shows that a graph with $n$ vertices has obstacle number at most $n^{2} / 2-n / 2$.

Open Question Is the obstacle number of a graph with $n$ vertices bounded above by a linear function of $n$ ?

Given a straight-edge drawing of $G$, let $G^{\prime}$ be the plane graph obtained by adding a vertex at every intersection of edges. We call a face of $G^{\prime}$ a face of the drawing of $G$. Every obstacle is contained in a face and may intersect as many as all of the nonedges through that face. Thus, the obstacle number of $G$ is the least number of faces needed in any drawing of $G$ to intersect all the non-edges. Also, given any connected set contained in a face of the drawing, we may replace it with a polygon intersecting the same non-edges. So relaxing the definition of obstacle to allow any connected set does not change the obstacle number.

\section{Graphs with Large Obstacle Number}

In this section, we construct graphs with obstacle number greater than 1 . We start by constructing a family of graphs with arbitrarily large obstacle number. Our construction uses the following theorem, first proven in [11]. 
Fig. 2 The graph $H$ formed by $v$ and a path from $a$ to $b$ in Case 1

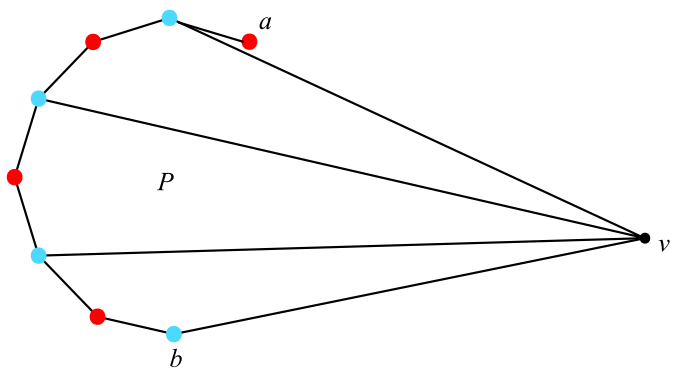

Theorem 1 (Happy Ending Theorem) For any integer $n \geq 3$, there exists a smallest positive integer $N(n)$ such that any set of at least $N(n)$ points in general position in the plane contains $n$ points that are the vertices of a convex $n$-gon.

More recently Tóth and Valtr proved $N(n) \leq\left(\begin{array}{c}2 n-5 \\ n-2\end{array}\right)+1[22]$. See $[1,2,18]$ for surveys of research on values of $N(n)$.

For positive integers $k$ and $m$, let $G_{k, m}$ be the graph consisting of the complete graph $K_{k}$, and for each subset $S$ containing exactly $2 m+2$ vertices of $K_{k}$, an additional vertex adjacent to the vertices in $S$.

Theorem 2 The obstacle number of $G_{k, m}$ is at least $m$ for sufficiently large $k$.

Proof Given a positive integer $m$, we use Theorem 1 to choose $k$ such that for any placement of $k$ vertices in general position in the plane, $4 m+4$ of them form the vertices of a convex $(4 m+4)$-gon. Suppose $(V, O)$ is an obstacle representation of $G_{k, m}$ for these values of $k$ and $m$. We show that $O$ contains at least $m$ obstacles.

Let $P$ be the convex $(4 m+4)$-gon in the vertices of $K_{k}$. Starting from an arbitrary vertex of $P$ and traversing $P$ counter-clockwise, we label every second vertex of $P$ blue and the remaining vertices red. Because there are $2 m+2$ blue vertices in $P$, there is a vertex $v$ of $G_{k, m}$ adjacent to all of the blue vertices but none of the red. We consider the placement of $v$ in the obstacle representation $(V, O)$.

Case 1. The point $v$ is outside $P$. There are two vertices $a$ and $b$ of $P$, such that the segments $\overline{v a}$ and $\overline{v b}$ lie on the boundary of the convex hull of $v \cup P$. There are two paths from $a$ to $b$ along the perimeter of $P$. Because there are $2 m+2$ blue vertices, one of these paths must contain at least $m+1$ of them. This path and its edges to $v$ form a subgraph $H$ of $G$ embedded in the plane without edge crossings, as shown in Fig. 2. Its faces are quadrilaterals whose vertices are $v$ and three consecutive vertices of $P$. Each quadrilateral $Q$ must contain an obstacle to block the non-edge between $v$ and the red vertex of $Q$. There are at least $m$ faces of $H$, so $G_{k, m}$ requires at least $m$ obstacles.

Case 2. The point $v$ is inside $P$. In this case, the edges from $v$ to the blue vertices of $P$ form $2 m+2$ disjoint quadrilaterals, as shown in Fig. 3. As in Case 1, each quadrilateral is composed of two blue vertices, a red vertex, and $v$, requiring at least $2 m+2$ obstacles to block the non-edges from the red vertices to $v$.

Because the representation $(V, O)$ requires at least $m$ obstacles in both cases, $G_{k, m}$ has obstacle number at least $m$. 
Fig. 3 The graph $H$ in Case 2

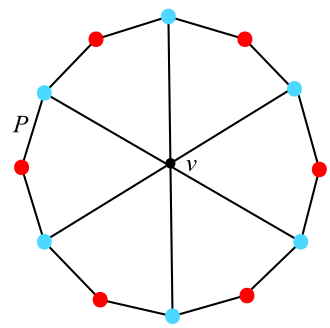

Fig. 4 The three ways to draw a 4-cycle in the plane
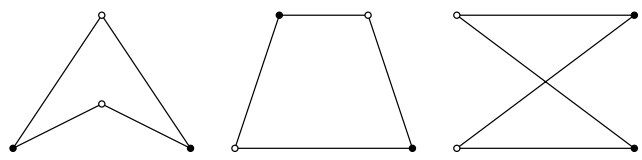

Note that by the upper bound $N(n) \leq\left(\begin{array}{c}2 n-5 \\ n-2\end{array}\right)+1$ given above, it suffices to choose $k=\left(\begin{array}{c}8 m+3 \\ 4 m+2\end{array}\right)+1$ in the proof of Theorem 2 , in which case the number of vertices in $G_{k, m}$ is

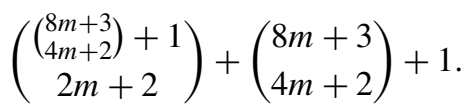

We expect that there are much smaller graphs with obstacle number $m$. The next theorem gives an example of a graph with 12 vertices with obstacle number 2 .

Given points $a, b$, and $c$ in the plane, we say that $a$ sees $b$ to the left of $c$ (equivalently, sees $c$ to the right of $b$ ) if the points $a, b$, and $c$ appear in clockwise order. If $a$ sees $b$ to the left of $c, c$ to the left of $d$, and $d$ to the left of $b$, then $a$ is in the convex hull of $b, c$, and $d$. Thus, if $a$ is outside the convex hull of some set $S$ of points, the relation " $a$ sees to the left of" is transitive on $S$, and hence is a total ordering of $S$, which we call the $a$-sight ordering of $S$.

Let $H$ be a complete bipartite graph with parts $R$ (colored red) and $B$ (colored blue), $|R| \geq 2$ and $|B| \geq 3$.

Lemma 3 In any obstacle representation of $H$ with one obstacle, the convex hull of $R$ is disjoint from the convex hull of $B$. Furthermore, the $r$-sight ordering of $B$ is the same for every red vertex $r$, and the $b$-sight ordering of $R$ is the same for every blue vertex $b$.

Proof Let $S$ be an obstacle representation of $H$ with one obstacle. There are three ways to draw a 4-cycle in the plane: the dart, the square, and the bowtie, shown in Fig. 4. No 4-cycle in $H$ can form a dart in $S$, because one non-edge appears inside the cycle and one appears outside, requiring two obstacles. If a 4-cycle in $H$ forms a square in $S$, then any other vertex of $H$ inside the square forms a dart with three vertices of the square, and any other vertex outside the square creates a non-edge outside the square in addition to the non-edges already inside. So every 4-cycle in $H$ must form a bowtie in $S$.

Suppose by way of contradiction that the convex hulls of $R$ and $B$ intersect. There are two ways this can happen. 

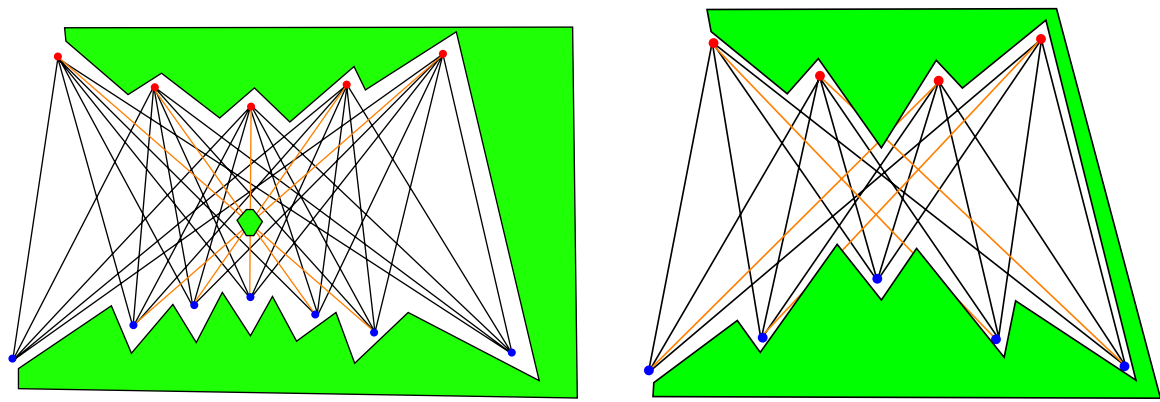

Fig. 5 A two-obstacle representation of $K_{5,7}^{*}$ and a one-obstacle representation of $K_{4,5}^{*}$

Case 1. $\overline{r_{1} r_{2}}$ and $\overline{b_{1} b_{2}}$ intersect for some red vertices $r_{1}$ and $r_{2}$ and some blue vertices $b_{1}$ and $b_{2}$. In this case, the 4-cycle $r_{1}, b_{1}, r_{2}, b_{2}$ forms a square.

Case 2. The convex hull of $R$ is completely contained in the convex hull of $B$. Let $r_{1}$ be a red vertex. Then every line through $r_{1}$ has blue vertices on both sides, so two blue vertices $b_{1}$ and $b_{2}$ are on different sides of the line through $r_{1}$ and any other red vertex $r_{2}$. These four vertices create a dart if the segments $\overline{r_{1} r_{2}}$ and $\overline{b_{1} b_{2}}$ do not intersect and a square if they do.

Because both cases lead to contradictions, the convex hulls of $R$ and $B$ are disjoint.

We now prove that the $r$-sight ordering of $B$ is the same for every red vertex $r$, and the $b$-sight ordering of $R$ is the same for every blue vertex $b$. Suppose the $r_{1}$-sight ordering of $B$ and the $r_{2}$-sight ordering of $B$ are different for some red vertices $r_{1}$ and $r_{2}$. Then for some two blue vertices $b_{1}$ and $b_{2}, r_{1}$ sees $b_{1}$ to the left of $b_{2}$, but $r_{2}$ sees $b_{2}$ to the left of $b_{1}$. Equivalently, $r_{1}$ and $r_{2}$ are on different sides of the line $\overleftrightarrow{b_{1} b_{2}}$. But that means these four vertices form a dart or a square, so in fact the sight orderings must be the same.

Let $K_{m, n}^{*}, m \leq n$, be the graph formed by deleting $m$ non-adjacent edges from the complete bipartite graph $K_{m, n}$. Note that $K_{m, n}^{*}$ has obstacle number at most 2, because we can arrange the $m$ non-adjacent non-edges to all intersect the same small obstacle. Figure 5 shows a two-obstacle representation of $K_{5,7}^{*}$. On the other hand, $K_{4,5}^{*}$ has obstacle number 1, also shown in Fig. 5. We conjecture that $K_{5,5}^{*}$ has obstacle number 2 .

Theorem $4 K_{5,7}^{*}$ has obstacle number exactly 2.

Proof It suffices to show that $K_{5,7}^{*}$ cannot be represented with one obstacle. Suppose by way of contradiction that there exists an obstacle representation of $K_{5,7}^{*}$ with a single obstacle. Let $R$ be the partite set of size 7, colored red, and $B$ be the partite set of size 5, colored blue. There are two red vertices $r_{1}$ and $r_{2}$ adjacent to all five blue vertices in $K_{5,7}^{*}$, inducing the subgraph $K_{2,5}$. By Lemma 3, the $r_{1}$-sight ordering of $B$ and the $r_{2}$-sight ordering of $B$ are the same. Let $b_{L}$ be the left-most blue vertex and $b_{R}$ be the right-most blue vertex in this common ordering.

For the remainder of the proof, we ignore the red vertices not adjacent to $b_{L}$ and $b_{R}$, respectively, only considering the five red vertices all adjacent to $b_{L}$ and $b_{R}$. We 
Fig. 6 The $b_{L^{-}}, b$-, and $b_{R}$-sight orderings of the red vertices are the same
Fig. 7 Non-edge $\overline{r^{*} b^{*}}$ requires a second obstacle
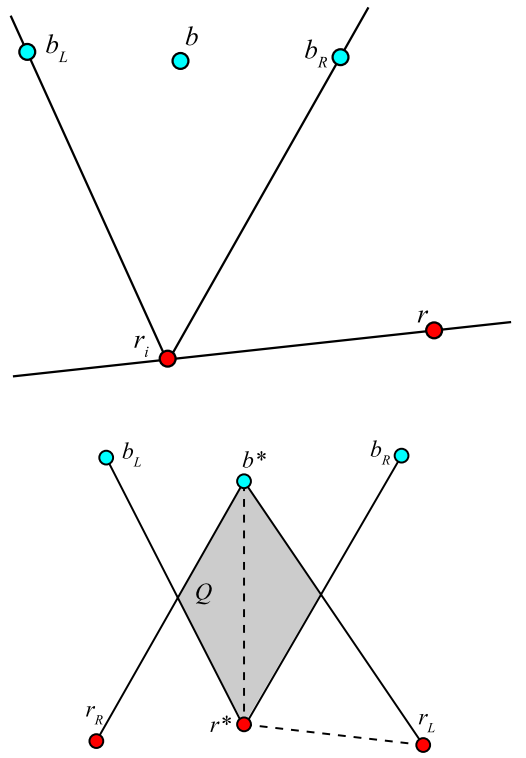

claim that every blue vertex has the same sight ordering of the five red vertices. By Lemma 3 , the $b_{L}$ - and $b_{R}$-sight orderings of the five red vertices are the same, and for any other blue vertex $b$ not adjacent to some red vertex $r$, the $b_{L^{-}}, b_{R^{-}}$, and $b$-sight orderings of the four red vertices other than $r$ are the same. To show that the $b$-sight ordering of all five vertices is the same as the $b_{L}$ - and $b_{R}$-sight orderings, we show that for any red vertex $r_{i}$ other than $r$, the $b$-sight ordering of $r_{i}$ and $r$ is the same as the $b_{L^{-}}$and $b_{R}$-sight ordering of $r_{i}$ and $r$.

Because $r_{i}$ is adjacent to $b_{L}, b$, and $b_{R}$, by Lemma 3 the $r_{i}$-sight ordering of those three blue vertices is the same as the $r_{1}$ - and $r_{2}$-sight ordering of those vertices; namely, $r_{i}$ sees $b$ between $b_{L}$ and $b_{R}$. So $b$ lies in the interior of the angle $\angle b_{L} r_{i} b_{R}$, as shown in Fig. 6. Because the $b_{L}$ - and $b_{R}$-sight orderings of $r_{i}$ and $r$ are the same, $b_{L}$ and $b_{R}$ are on the same side of the line $\overleftrightarrow{r_{i} r}$, so $b$ must be too. Thus the $b$-sight ordering of the five red vertices must be the same as the $b_{L}$-and $b_{R}$-sight ordering of these vertices.

Three red vertices-all except $r_{1}$ and $r_{2}$ - have blue non-neighbors. In the common blue-sight-ordering of the five red vertices, some red vertex $r^{*}$ not adjacent to some blue vertex $b^{*}$ is neither the left-most red vertex $r_{L}$ nor the right-most red vertex $r_{R}$. Then the non-edge $\overline{r^{*} b^{*}}$ is completely contained in the intersection of the interiors of angles $\angle b_{L} r^{*} b_{R}$ and $\angle r_{L} b^{*} r_{R}$, as shown in Fig. 7. This region is a quadrilateral, which we call $Q$. The vertices $r_{L}, r_{R}, b_{L}$, and $b_{R}$ lie outside $Q$, because, for instance, if $r_{L}$ were interior to angle $\angle b_{L} r^{*} b_{R}$, then $b_{L}$ and $b_{R}$ would be on different sides of the line $\overleftrightarrow{r_{L} r^{*}}$, and so the $b_{L}$ - and $b_{R}$-sight orderings of $r_{L}$ and $r^{*}$ would be different. Similar reasoning shows that all four points $r_{L}, r_{R}, b_{L}$, and $b_{R}$ are outside $Q$, and so $Q$ is bounded by the edges $\overline{r^{*} b_{L}}, \overline{r^{*} b_{R}}, \overline{b^{*} r_{L}}$, and $\overline{b^{*} r_{R}}$ and contains the non-edge $\overline{r^{*} b^{*}}$ but excludes the non-edge $\overline{r^{*} r_{L}}$. Hence there must be an obstacle in $Q$ and an obstacle outside $Q$, so $K_{5,7}^{*}$ has obstacle number 2 . 
Fig. 8 A non-visibility graph with obstacle number 1

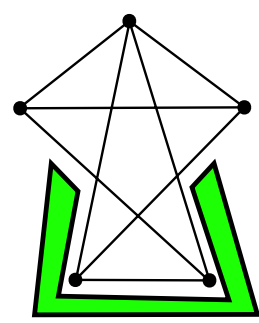

Open Question For an integer $m>1$, what is the smallest number of vertices of a graph with obstacle number $m$ ?

Open Question For each $m \geq 3$, is there a graph with obstacle number exactly $m$ ?

Open Question Are there bipartite graphs with arbitrarily large obstacle number?

\section{Graphs with Obstacle Number 1}

We define the outside of a drawing $D$ to be the outside face of $D$. The inside of $D$ is the complement of the outside.

Each of our examples of graphs with obstacle number 1 has an obstacle representation with the single obstacle contained in the outside of the drawing. We call such a representation an outside-obstacle representation.

Open Question Does every graph with obstacle number 1 have an outside-obstacle representation?

One family of graphs with obstacle number 1 is the family of polygon-vertex visibility graphs. A polygon-vertex visibility graph is the graph formed by taking the vertices of a polygon in the plane and drawing an edge between two vertices if the line segment between them has no point exterior to the polygon [19]. In such a drawing, every non-edge intersects the outside face, so one obstacle is sufficient. However, the converse is not true. The graph shown in Fig. 8 has obstacle number 1 but is not a polygon-vertex visibility graph [19], because in every outside-obstacle representation, the polygon bounding the inside of the drawing has vertices that are not vertices of the graph.

Recall that a graph is outerplanar if it has a planar embedding with all vertices on the outside face. In [10], ElGindy proved that every maximal outerplanar graph is a polygon-vertex visibility graph, which implies that every maximal outerplanar graph has obstacle number 1 . We now extend this result to show that all outerplanar graphs have obstacle number 1 .

Theorem 5 Every outerplanar graph has obstacle number 1. In particular, every outerplanar graph has an outside-obstacle representation. 
Fig. 9 Adding the vertices $v_{1}, \ldots, v_{k}$

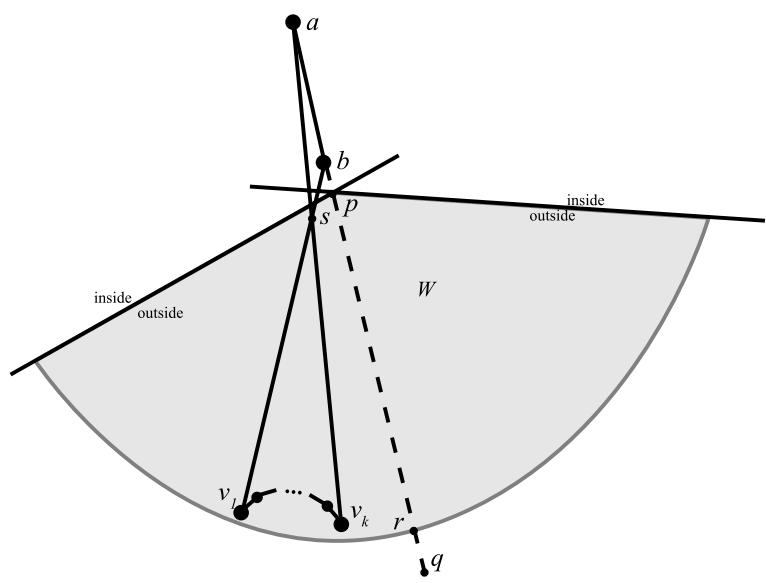

Proof Let $G$ be an outerplanar graph. If $G$ is disconnected, and each component of $G$ has an outside-obstacle representation, we can place these representations side by side and connect the obstacles in the outside face to form a single obstacle. Thus, we may assume that $G$ is connected. We use induction on the number of vertices of $G$. If $G$ consists of a single vertex, the statement follows easily. Assume that $G$ has $n>1$ vertices.

Case 1. Every vertex of $G$ has degree at least 2 . We find a cycle $F$ of $G$ with consecutive adjacent vertices $a, b, v_{1}, \ldots, v_{k}$, such that the vertices $v_{1}, \ldots, v_{k}$ all have degree 2 in $G$, in the following way. Let $B$ be a 2-connected component of $G$ that contains at most one cut vertex of $G$. Then let $F$ be a face of $G$ corresponding to any leaf of the weak dual of $B$ [25].

Let $H$ be the graph obtained by deleting $v_{1}, \ldots, v_{k}$ from $G . H$ is a connected outerplanar graph, so by the inductive hypothesis, $H$ has an outside-obstacle representation $R$ with a single obstacle $O$. We add $v_{1}, \ldots, v_{k}$ to $R$ in the following way, as shown in Fig. 9.

In $R$, find edge $\{a, b\}$ and follow ray $\overrightarrow{a b}$ to where it first intersects the outside of the drawing. Let $p$ and $q$ be points on $\overrightarrow{a b}$ such that closed line segment $\overline{a p}$ is inside the drawing of $H$ and the open line segment $\overline{p q}$ is outside the drawing. The points $p$ and $b$ may be identical.

There exists $\varepsilon>0$ such that the distance between $O$ and the inside of $H$ is at least $\varepsilon$, and such that $p$ is at least distance $\varepsilon$ away from every edge of $H$ not incident with $p$. Choose $r \in \overline{p q}$ such that the distance between $p$ and $r$ is less than $\varepsilon$, and draw a circle $C$ through $r$ centered at $p$. Then because $r$ is in the outside of the drawing, there is some circular wedge $W$ of $C$ that contains $r$ and lies completely outside the drawing. We place $v_{1}, \ldots, v_{k}$ in $W$ in the following way.

Case 1a. $k=1$. Place $v_{1}$ in $W$ close enough to $r$ that for every vertex $x$ of $H$, $v_{1}$ and $r$ are on the same side of the lines $\overleftrightarrow{a x}$ and $\overleftrightarrow{b x}$. Then the $v_{1}$ - and $r$-sight orderings of $a$ and $x$ are the same, and similarly with $b$ and $x$. Because $r, a$, and $b$ are collinear, $r$ sees $x$ on the same side of $b$ as of $a$, so $v_{1}$ does, too. Thus, $v_{1}$ sees no vertex between $a$ and $b$, and $a$ and $b$ appear consecutively in the $v_{1}$-sight ordering of the vertices of $H$. 
To place the obstacle in the representation, it suffices to show that every non-edge incident to $v_{1}$ intersects the outside of the drawing. Note that the inside of $G$ is the union of triangle $\triangle a b v_{1}$ with the inside of $H$. Because every non-edge incident to $v_{1}$ intersects the outside of $H$ and is disjoint from triangle $\triangle a b v_{1}$, it intersects the outside of $G$.

Case $1 b . k \neq 1$. Place $v_{1}$ and $v_{k}$ in $W$ in such a way that edges $\overline{a v_{k}}$ and $\overline{b v_{1}}$ intersect at point $s \in W$. Place $v_{2}, \ldots, v_{k-1}$ inside triangle $\Delta v_{1} v_{k} s$, such that $v_{1}, \ldots, v_{k}$ are the vertices of a convex $k$-gon, in that order, as shown in Fig. 9. We also place these vertices so that no non-edge of $G$ contains the point $s$.

Now we place the obstacle in the representation. We will show that every non-edge of $G$ intersects the outside of the drawing of $G$. Then we can easily place an outside obstacle blocking all non-edges. There are three types of non-edge to consider: if $x$ and $y$ are arbitrary vertices of $H$, and $1 \leq i, j \leq k$, then we have potential non-edges of the form $\{x, y\},\left\{x, v_{i}\right\}$, and $\left\{v_{i}, v_{j}\right\}$. First, consider a non-edge of the form $\{x, y\}$. The segment $\overline{x y}$ intersects the obstacle $O$ because $\{x, y\}$ is a non-edge of $H$, and $O$ is still outside $G$ because $W$ is disjoint from $O$. Now consider a non-edge of the form $\left\{x, v_{i}\right\}$. Note that the removal of the point $s$ would separate the inside of the drawing of $G$ into two regions; by construction, $\overline{x v_{i}}$ does not contain $s$, so it intersects the outside. Finally, consider a non-edge of the form $\left\{v_{i}, v_{j}\right\}$. By construction, $v_{i} v_{j}$ is completely contained in the outside of the representation.

Case 2. $G$ has a vertex $v$ of degree 1 . We remove $v$ to form $H$, an outerplanar subgraph of $G$ which by the inductive hypothesis has an outside-obstacle representation. Let $a$ be the one neighbor of $v$, and let $b$ be any neighbor of $a$. (If $a$ has no neighbor, then $G$ is a single edge and is trivially an outside-obstacle graph.) Then, as before, use the outside-obstacle representation of $H$ to find point $p$ and wedge $W$. Place $v$ in $W$; all non-edges incident to $v$ intersect the outside of $G$.

Thus, every outerplanar graph $G$ is an outside-obstacle graph. In particular, every outerplanar graph has obstacle number 1 .

Open Question Does every planar graph have obstacle number 1? Specifically, what are the obstacle numbers of the icosahedron and the dodecahedron graphs?

\section{Graphs with Large Convex Obstacle Number}

The convex obstacle number of a graph $G$ is the least number of obstacles in any obstacle representation of $G$ with all obstacles convex. Note that for a given graph $G$, the convex obstacle number of $G$ is always greater than or equal to the obstacle number of $G$. In this section, we describe a family of graphs for which the obstacle number is 1 and the convex obstacle number is arbitrarily large.

Recall that the Ramsey number $R_{k}(3)=R(3,3, \ldots, 3)$ (where the number 3 is repeated $k$ times) is the least integer $m$ such that any coloring of the edges of the complete graph $K_{m}$ with $k$ colors contains a monochromatic triangle [20]. Choose integers $a_{1}, a_{2}, \ldots, a_{n}$ with $a_{1} \geq 2$ and $a_{k} \geq R_{k-1}$ (3) for $2 \leq k \leq n$.

Consider the complete $n$-partite graph $K_{a_{1}, a_{2}, \ldots, a_{n}}$. We describe a drawing of this graph that requires one regular obstacle or $n$ convex obstacles. For $1 \leq i \leq n$, select 
Fig. 10 Placing the $i$ th partite set of $K_{a_{1}, a_{2}, \ldots, a_{n}}$

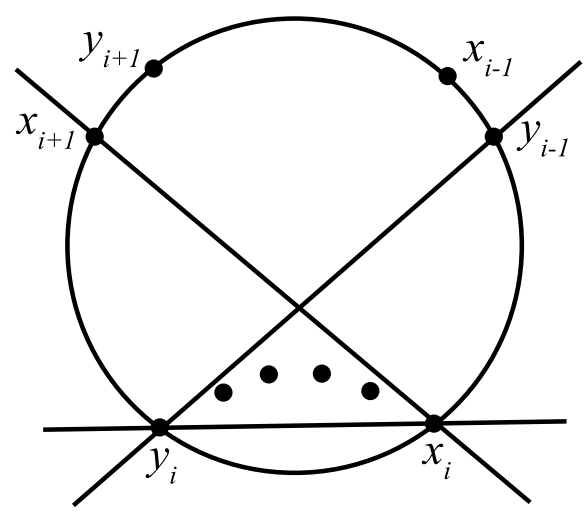

two vertices $x_{i}$ and $y_{i}$ from the $i$ th partite set, and position them on a circle $C$, in the order $x_{1}, y_{1}, x_{2}, y_{2}, \ldots, x_{n}, y_{n}$ clockwise. Then place the other vertices of the $i$ th partite set inside the triangle enclosed by lines $\overleftrightarrow{x_{i} y_{i}}, \overleftrightarrow{x_{i} x_{i+1}}$, and $\overleftrightarrow{y_{i} y_{i-1}}$, such that all $a_{i}$ vertices form a convex polygon, as in Fig. 10. This $i$ th convex polygon contains all non-edges of the $i$ th partite set, but intersects no edges, so we can place one convex obstacle per partite set, for a total of $n$ convex obstacles. Connecting these convex obstacles around the outside would produce a single connected non-convex obstacle, so $K_{a_{1}, a_{2}, \ldots, a_{n}}$ has obstacle number 1 and convex obstacle number at most $n$.

Theorem 6 The complete $n$-partite graph $K_{a_{1}, a_{2}, \ldots, a_{n}}$ has convex obstacle number exactly $n$.

Proof We use induction on $n$. When $n=1, K_{a_{1}, a_{2}, \ldots, a_{n}}$ is the graph consisting of two disconnected vertices, and clearly requires one obstacle. Suppose for the sake of contradiction that there is an obstacle representation of $K_{a_{1}, a_{2}, \ldots, a_{n}}$ with only $n-1$ convex obstacles. Label these obstacles with $n-1$ different colors. We color each non-edge in the $n$th partite set with the color corresponding to an obstacle that intersects that non-edge. The choice of $a_{n}$ guarantees a triangle of non-edges all blocked by the same obstacle. This obstacle must intersect a non-edge in the smaller graph $K_{a_{1}, a_{2}, \ldots, a_{n-1}}$; if not, we could represent $K_{a_{1}, a_{2}, \ldots, a_{n-1}}$ with $n-2$ obstacles, contradicting the inductive hypothesis. The two vertices of this old non-edge and the three vertices of the triangle of new non-edges induce a $K_{2,3}$, and all of its non-edges are blocked by the same obstacle. But we show below in Corollary 9 that $K_{2,3}$ has convex obstacle number 2 , which is a contradiction. Thus, $K_{a_{1}, a_{2}, \ldots, a_{n}}$ has convex obstacle number exactly $n$.

By Theorem $6, K_{a_{1}, a_{2}, \ldots, a_{n}}$ is an example of a graph with obstacle number 1 and arbitrarily large convex obstacle number. On the other hand, the convex obstacle number of any graph is greater than or equal to its obstacle number, so the graphs $G_{k, m}$ in Theorem 2 have both large obstacle number and large convex obstacle number.

Open Question Are there planar graphs or outerplanar graphs with arbitrarily large convex obstacle number? 


\section{Graphs with Convex Obstacle Number 1}

In this section, we completely characterize graphs with convex obstacle number 1.

A graph $G$ is a circular arc graph if there exists a one-to-one correspondence between the set of vertices of $G$ and a set of arcs of a circle, such that two arcs intersect if and only if the corresponding vertices are adjacent. The set of arcs is called a circular arc representation of $G[8,13,17,24]$. We assume without loss of generality that every circular arc representation is on a unit circle and the endpoints of arcs are distinct. We define a number of special properties of circular arc representations and graphs. We say that $R$ is non-double-covering if no two $\operatorname{arcs}$ in $R$ cover the entire circle. A graph is a non-double-covering circular arc graph if it has a nondouble-covering circular arc representation. Fischer and Simon call such a circular arc representation not inherently 2-covered [12]. McConnell calls two arcs of this form double overlapping $[9,16]$. These representations also appear in Golumbic and in Hell and Huang without a name $[13,15]$. We say that $R$ is bounded if every arc in $R$ has length less than $\pi$.

Consider the sequence of endpoints of arcs in $R$, traversing the circle counterclockwise. For an arc $A$ of $R$, its endpoint further counter-clockwise is its left endpoint, and its endpoint further clockwise is its right endpoint. We say that $R$ is alternating if the sequence of endpoints of arcs in $R$ alternates between left endpoints and right endpoints of arcs.

If a circular arc representation $R$ of a graph $G$ has the property that no arc is properly contained in another, then $R$ is proper and $G$ is a proper circular arc graph [8, $23]$. Note also that every proper circular arc graph has a non-double-covering circular arc representation [13]. We prove in Theorem 8 that every non-double-covering circular arc graph has convex obstacle number 1, so every proper circular arc graph has convex obstacle number 1 . The converse is not true: $K_{1,3}$ is easily seen to have convex obstacle number 1 but is not a proper circular arc graph.

In [12], the authors (using different terminology) show that every graph with convex obstacle number 1 is a non-double-covering circular arc graph. They also claim that there are non-double-covering circular arc graphs that have convex obstacle number greater than 1. By contrast, we prove in Theorem 8 that a graph has convex obstacle number 1 if and only if it is a non-double-covering circular arc graph. We include both directions of the proof for completeness, and to provide additional details omitted in [12].

Lemma 7 Suppose $G$ is a circular arc graph. $G$ has a non-double-covering circular arc representation if and only if $G$ has a bounded circular arc representation.

Proof Suppose $G$ is a circular arc graph with circular arc representation $R$. Note that if every arc in $R$ has length less than $\pi$, then every two $\operatorname{arcs}$ in $R$ must have total length less than $2 \pi$, so $R$ is non-double-covering. It remains to show that if $R$ is non-double-covering, then $G$ has a bounded circular arc representation.

Specifically, we use induction on the number of vertices of $G$ to show that $G$ has a bounded circular arc representation in which the arc endpoints appear in the same 
order as in the non-double-covering representation $R$. If $G$ has only one vertex, then $G$ can be represented by one arc of length less than $\pi$. Suppose $G$ has $n$ vertices and non-double-covering circular arc representation $R$.

Case 1. Some arc $A$ is properly contained in another $\operatorname{arc} B$ of $R$. Let $a$ be the vertex of $G$ corresponding to arc $A$. By the inductive hypothesis, there is a bounded representation $R^{\prime}$ of $G-a$ containing an arc $X^{\prime}$ for every arc $X \in R-A$, such that the endpoints of arcs in $R^{\prime}$ are in the same order as the corresponding endpoints in $R-A$. Choose any arc $A^{\prime}$ such that when we add $A^{\prime}$ to $R^{\prime}$, the arc endpoints in $R^{\prime}$ are in the same order as in $R$. Then $A^{\prime} \subset B^{\prime}$, so length $\left(A^{\prime}\right)<\operatorname{length}\left(B^{\prime}\right)<\pi$, and $R^{\prime}$ is bounded after the addition of $A^{\prime}$.

Case 2. $R$ is proper and not alternating. Because $R$ has the same number of left and right endpoints, in this case there exist two $\operatorname{arcs} A$ and $B$ in $R$ such that the left endpoints of $A$ and $B$ occur consecutively in the sequence of endpoints of $R$. Say the left endpoint of $A$ occurs further counter-clockwise. Again we find a bounded representation $R^{\prime}$ of $G-a$ by the inductive hypothesis, containing an arc $X^{\prime}$ for every arc $X \in R-A$, with the endpoints of $R^{\prime}$ in the same order as in $R-A$. First, we place the right endpoint of $A^{\prime}$ between the same arc endpoints in $R^{\prime}$ as in $R$. Because $R$ is proper, this right endpoint of $A^{\prime}$ is contained in $B^{\prime}$. The arc from the left endpoint of $B^{\prime}$ to the right endpoint of $A^{\prime}$ is shorter than $B^{\prime}$, so by choosing the left endpoint of $A^{\prime}$ close enough to the left endpoint of $B^{\prime}$, we may draw $A^{\prime}$ with length $\left(A^{\prime}\right)<$ length $\left(B^{\prime}\right)<\pi$.

Case 3. $R$ is proper and alternating. Let $A$ and $B$ be two $\operatorname{arcs}$ in $R$. If $A$ and $B$ intersect, then $A$ and $B$ contain the same number of arc endpoints, as follows. Without loss of generality, the left endpoint of $B$ is contained in $A$. Then because $R$ is proper, for every left endpoint in $A \backslash B$ of some other arc $C$, the right endpoint of $C$ must lie in $B \backslash A$. So the number of left endpoints in $A$ equals the number of the right endpoints in $B$; because $R$ is alternating, each interval has the same number of left endpoints as right endpoints, so $A$ and $B$ must contain the same total number of endpoints. Notice that as a point moves around the circle in $R$, the number of arcs containing the point alternates between $k$ and $k-1$ as the point passes left and right endpoints. So if $R$ is disconnected, it is completely disconnected. Whether $R$ is connected or completely disconnected, we may conclude that every arc in $R$ contains the same number of endpoints.

We form a new representation $R^{\prime}$ of $G$ by evenly spacing the endpoints of $\operatorname{arcs}$ in $R$ around the circle, so that every arc in $R^{\prime}$ has the same length. If this length is less than $\pi$, then $R^{\prime}$ is bounded. If every arc has length exactly $\pi$, then move each endpoint slightly (say, by $\varepsilon<2 \pi / 4 n$, if there are $2 n$ endpoints) to shorten each arc and make a bounded representation $R^{\prime \prime}$. If every arc has length greater than $\pi$, consider any arc $A \in R^{\prime}$ with left endpoint $a_{1}$ and right endpoint $a_{2}$. The point $p$ antipodal to $a_{1}$ is an endpoint of $R^{\prime}$, because $R^{\prime}$ has an even number of endpoints. Between $p$ and $a_{2}$ is the endpoint in $A$ closest to $a_{2}$; this closest endpoint is the left endpoint $b_{1}$ of some arc $B$ because $R^{\prime}$ is alternating. Then, because $B$ has length greater than $\pi, B$ must contain $a_{1}$, in which case $A \cup B$ is the whole circle, contradicting the assumption that $R$, and therefore $R^{\prime}$, is non-double-covering. Thus, no arc has length greater than $\pi$, and so either $R^{\prime}$ or $R^{\prime \prime}$ is a bounded representation of $G$. 

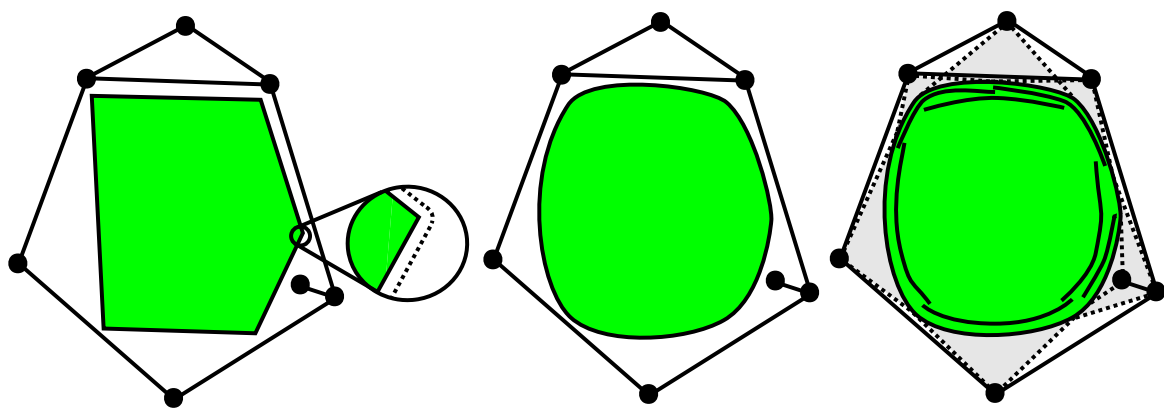

Fig. 11 Transforming an obstacle representation into a circular arc representation

Theorem 8 A graph $G$ has convex obstacle number 1 if and only if it is a non-doublecovering circular arc graph.

Proof First, suppose that $G$ has an obstacle representation with one convex obstacle $P$. We will construct a non-double-covering circular arc representation of $G$ with open arcs with endpoints not necessarily distinct. In any such representation, for any two arcs with the same endpoint, one arc can be shortened slightly to distinguish the endpoints, and then the arcs can be changed to closed arcs without losing the non-double-covering property. Thus, even though we use open $\operatorname{arcs,} G$ also has a non-double-covering representation with the more conventional closed arcs.

The process is summarized in Fig. 11. Choose $\varepsilon>0$ such that $\varepsilon$ is less than the distance from $P$ to any edge of $G$. Let $C$ be the set of points of distance at most $\varepsilon$ away from $P$. Then $C$ is convex and blocks exactly the non-edges of $G$, and its boundary is differentiable.

There is a well-defined tangent line $L(x)$ at every point $x$ of the boundary of $C$. $L(x)$ determines an open half-plane $H(x)$ disjoint from $C$. For any vertex $v$ of $G$, if $H(x)$ contains $v$, then $L(x)$ separates $v$ from $C$ [12]. Let $A(v)$ be the set of such $x$. Notice that if $L\left(x_{1}\right)$ separates $v$ from $C$ and $L\left(x_{2}\right)$ does not, then there must be some point $x^{*}$ between $x_{1}$ and $x_{2}$ with $v \in L\left(x^{*}\right)$. Thus, the two such $x^{*}$ on the boundary of $C$ are endpoints of $A(v)$, which is the open arc of $C$ on the side nearest to $v$.

We claim that for any two vertices $v_{1}$ and $v_{2}$ of $G$, their corresponding $\operatorname{arcs} A\left(v_{1}\right)$ and $A\left(v_{2}\right)$ intersect if and only if $v_{1} \sim v_{2}$ in $G$. It suffices to show that $A\left(v_{1}\right)$ and $A\left(v_{2}\right)$ intersect if and only if $\overline{v_{1} v_{2}} \cap C=\emptyset$. For the forward direction, consider $x \in$ $A\left(v_{1}\right) \cap A\left(v_{2}\right)$. Then $v_{1}, v_{2} \in H(x)$, so $\overline{v_{1} v_{2}} \in H(x)$ and is disjoint from $C$. For the reverse direction, suppose $\overline{v_{1} v_{2}} \cap C=\emptyset$. Because $\overline{v_{1} v_{2}}$ and $C$ are disjoint convex sets, there is some line $L$ separating them. Without loss of generality, take $L$ to be the $x$-axis, $\overline{v_{1} v_{2}}$ to be below the axis, and $C$ to be above. Let $y_{0}$ be the least $y$ coordinate in $C$, and let $L^{\prime}$ be the horizontal line with equation $y=y_{0}$. Then $L^{\prime}$ is tangent to $C$ at some point $x$, with $v_{1}$ and $v_{2}$ below $L^{\prime}$ and $C$ above, so $x \in A\left(v_{1}\right) \cap A\left(v_{2}\right)$.

Let $R=\{A(v) \mid v \in G\}$, and let $\varphi$ be any homeomorphism from the boundary of $C$ to a circle. The set of $\operatorname{arcs} \varphi(R)=\{\varphi(A(v)) \mid v \in G\}$ forms a circular arc representation of $G$. Additionally, $\varphi(R)$ is non-double-covering, as follows. It suffices to show that $R$ is non-double-covering, because this property will be preserved under a 
Fig. 12 Replacing the circle $C$ with the polygon $P$

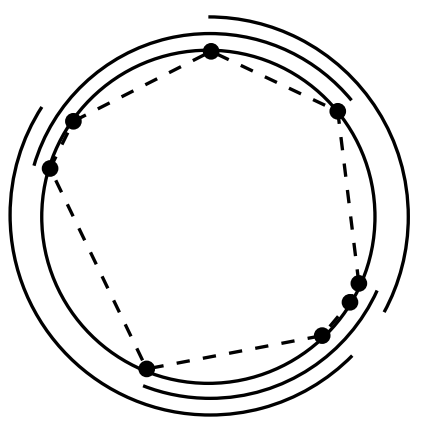

Fig. 13 If segment $\overline{v_{1} v_{2}}$ is blocked by $C$, it is blocked by $P$

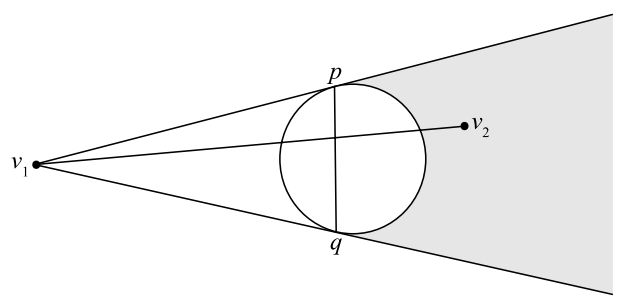

homeomorphism. Suppose by way of contradiction that there are two $\operatorname{arcs} A\left(v_{1}\right)$ and $A\left(v_{2}\right)$ whose union is the entire boundary of $C$. Consider the set of all tangent lines to $C$. This set has exactly two lines of every slope (including infinite slope). Then because $A\left(v_{1}\right)$ and $A\left(v_{2}\right)$ intersect, by the Pigeonhole principle one of them (say $A\left(v_{1}\right)$ ) contains two points $x_{1}$ and $x_{2}$ with corresponding parallel tangent lines $L\left(x_{1}\right)$ and $L\left(x_{2}\right)$. Therefore, $v_{1} \in H\left(x_{1}\right) \cap H\left(x_{2}\right)$, which is impossible because $H\left(x_{1}\right)$ and $H\left(x_{2}\right)$ are disjoint. Therefore, $R$ is non-double-covering.

Now suppose that $G$ is a non-double-covering circular arc graph. Let $S$ be a bounded circular arc representation of $G$ on circle $C$. For each arc $A$ of $S$, let $T_{1}$ and $T_{2}$ be the tangents to $C$ at the endpoints of $A$, and place a corresponding vertex $v$ at the intersection of $T_{1}$ and $T_{2}$. Note that, using the terminology from the first half of the proof, $A=A(v)$. Now let $P$ be the convex polygon with a vertex for every endpoint of every arc in $S$, as in Fig. 12.

We claim that these vertices together with obstacle $P$ form a convex obstacle representation of $G$. By the arguments above, given two vertices $v_{1}$ and $v_{2}$ of $G$, $A\left(v_{1}\right)$ and $A\left(v_{2}\right)$ intersect if and only if $\overline{v_{1} v_{2}}$ intersects the circle $C$. We show this is still true when $C$ is replaced with $P$. Because $P$ is a subset of $C$, any segment not intersecting $C$ does not intersect $P$. Now suppose $\overline{v_{1} v_{2}}$ intersects $C$. Let $v_{1}$ be a vertex of $G$, and let $p$ and $q$ be the endpoints of $A\left(v_{1}\right)$. The set of points $v_{2}$ outside $C$ for which $\overline{v_{1} v_{2}}$ intersects $C$ is the same as the set of points $v_{2}$ outside $C$ for which $\overline{v_{1} v_{2}}$ intersects $\overline{p q}$, as shown in Fig. 13. The segment $\overline{p q}$ is contained in $P$, so $\overline{v_{1} v_{2}}$ intersects $P$ whenever it intersects $C$.

Corollary 9 The convex obstacle number of $K_{2,3}$ is 2. 
Proof The convex obstacle number of $K_{2,3}$ is at most 2, because one obstacle for each partite set suffices. Then by Theorem 8, it suffices to show that $K_{2,3}$ is not a circular arc graph. Assume to the contrary that $R$ is a circular arc representation of $K_{2,3}$, with $A(v)$ denoting the arc corresponding to each vertex $v$. Let $\left\{a_{1}, a_{2}, a_{3}\right\}$ and $\left\{b_{1}, b_{2}\right\}$ be the two partite sets. Then $A\left(a_{1}\right), A\left(a_{2}\right)$, and $A\left(a_{3}\right)$ are disjoint, and however $A\left(b_{1}\right)$ is placed to intersect all three, it must contain one of them; without loss of generality, $A\left(a_{1}\right) \subset A\left(b_{1}\right)$. Then there is no way for $A\left(b_{2}\right)$ to intersect $A\left(a_{1}\right)$ but not $A\left(b_{1}\right)$, so $R$ is not a circular arc representation of $K_{2,3}$, and $K_{2,3}$ requires more than one convex obstacle.

Note that recent work has been done in recognizing circular arc graphs. McConnell provides a linear-time algorithm for recognizing circular arc graphs [16], Deng, Hell, and Huang provide a linear-time algorithm for recognizing proper circular arc graphs [8], and Durán et al. provide an $n^{2}$-time algorithm for recognizing unit circular arc graphs [9]. Since we have shown that the graphs with convex obstacle number 1 are exactly the non-double-covering circular arc graphs, we are interested in the recognition of these graphs as well.

Open Question With what time complexity can we recognize graphs with convex obstacle number 1 ?

\section{Graphs Representable with a $\boldsymbol{k}$-gon Convex Obstacle}

In this section, we characterize the graphs with a convex obstacle representation with one convex obstacle with $k$ sides. Recall that the clique covering number of a graph $G$ is the least number of cliques of $G$ which contain all of its vertices [14, 25]. Note that the clique covering number of $G$ is the chromatic number of $G^{C}$, the complement of $G$. For brevity, in the following discussion a $k$-gon representation of $G$ is an obstacle representation with one convex $k$-gon obstacle.

Theorem 10 A graph $G$ has a $k$-gon representation if and only if $G$ is a non-doublecovering circular arc graph that can be covered by $k$ cliques, for any $k \geq 2$.

In other words, the least number of sides of the obstacle in a convex obstacle representation of $G$ is equal to the clique covering number of $G$. For the forward direction, note that if $G$ has a $k$-gon representation, then the set of vertices visible from each edge of the $k$-gon forms a clique, so $G$ has clique covering number at most $k$. We prove the other direction of Theorem 10 as a series of lemmas. We first address the case $k \geq 3$, and then modify the proof for the case $k=2$, where the obstacle is a single segment.

A transversal of a circular arc representation $R$ is a set of points $T$ such that every arc of $R$ contains some point of $T$ [12]. We assume as before that all endpoints of $R$ are distinct, and thus we may also assume, without loss of generality, that no point in $T$ is an endpoint of an arc in $R$. We define a bounded $k$-points representation, $k \geq 3$, of a graph $G$ to be a bounded circular arc representation of $G$ together with 
Fig. 14 Placing the vertex $v_{i}$ at point $\varphi\left(x_{i}\right)$

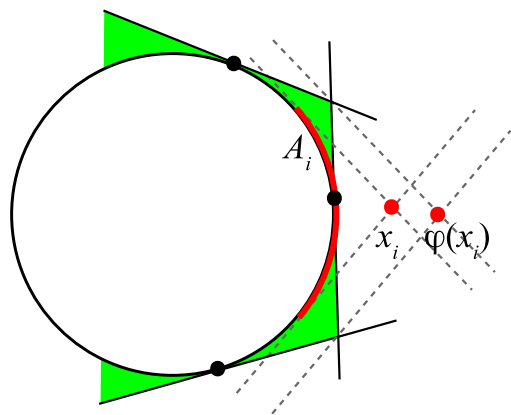

a transversal $T,|T|=k$, such that no half of the circle contains $T$. Theorem 12 and Lemmas 13,14 , and 15 construct a bounded $k$-points representation for any $k$-cliquecoverable non-double-covering circular arc graph. Lemma 11 completes the proof by transforming the bounded $k$-points representation into a $k$-gon representation.

Lemma 11 If a graph $G$ has a bounded $k$-points representation, $k \geq 3$, then $G$ has a k-gon representation.

Proof Let $R$ be a bounded $k$-points representation of $G$ on circle $C$. We construct a $k$-gon representation of $G$. Let $v_{1}, v_{2}, \ldots, v_{n}$ be the vertices of $G$, and for each $v_{i}$, let $A_{i}$ denote its corresponding arc in $R$. For each arc $A_{i}$, let $x_{i}$ be the intersection of the tangents to $C$ through the endpoints of $A_{i}$, as shown in Fig. 14. Then by the proof of Theorem 8, the set $\left\{x_{i}\right\}$ with obstacle $C$ is an obstacle representation of $G$.

Let $P$ be the convex $k$-gon enclosed by the $k$ lines tangent to $C$ at the points of the transversal $T . P$ is indicated by the shaded region shown in Fig. 14. The fact that not all points of $T$ are on the same half of $C$ guarantees that $P$ is in fact a bounded polygon. We define a function $\varphi$ on the set $\left\{x_{i}\right\}$ and prove that $\left\{\varphi\left(x_{i}\right)\right\}$ with obstacle $P$ is the desired $k$-gon representation of $G$.

Let us say a line $L$ is tangent to the polygon $P$ if $L \cap P$ is non-empty but $P$ is contained in one of the closed half-planes determined by $L$. The lines tangent to $P$ and the lines tangent to $C$ correspond according to slope: each line $L$ tangent to $C$ corresponds to the line $\Phi(L)$ parallel to $L$ and tangent to $P$, such that the closed half-plane determined by $L$ containing $C$ can be translated to coincide with the closed half-plane determined by $\Phi(L)$ containing $P$. Now since $x_{i}$ is the intersection of two tangents $L_{1}$ and $L_{2}$ to $C$, we may define $\varphi\left(x_{i}\right)$ to be the intersection of the corresponding tangents $\Phi\left(L_{1}\right)$ and $\Phi\left(L_{2}\right)$ to $P$. Each $\varphi\left(x_{i}\right)$ is external to $P$, as follows. By the construction of $P, \Phi\left(L_{1}\right)$ and $\Phi\left(L_{2}\right)$ contain the same vertex of $P$ if and only if $L_{1}$ and $L_{2}$ are tangent to $C$ at two points that lie between consecutive points of the transversal. This situation does not occur, because each $A_{i}$ contains a point of the transversal, so $\Phi\left(L_{1}\right)$ and $\Phi\left(L_{2}\right)$ intersect at $\varphi\left(x_{i}\right)$ external to $P$.

We now show that the set $\left\{\varphi\left(x_{i}\right)\right\}$ with obstacle $P$ is an obstacle representation of $G$. The key observation is that for a point $\varphi\left(x_{i}\right)$ and any line $\Phi(L)$ tangent to $P$, $\Phi(L)$ separates $\varphi\left(x_{i}\right)$ from $P$ if and only if $L$ separates $x_{i}$ from $C$. As in the proof of Theorem $8, \overline{\varphi\left(x_{i}\right) \varphi\left(x_{j}\right)}$ is disjoint from $P$ if and only if there is some line tangent to $P$ separating both $\varphi\left(x_{i}\right)$ and $\varphi\left(x_{j}\right)$ from $P$, just as $\overline{x_{i} x_{j}}$ is disjoint from $C$ if and only 
Fig. 15 The impossible arc $B$

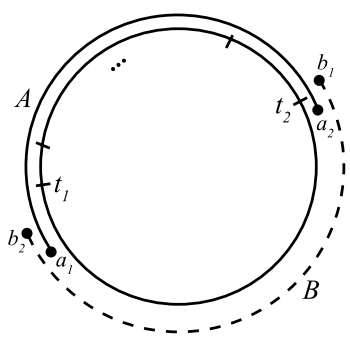

if there is some line tangent to $C$ separating both $x_{i}$ and $x_{j}$ from $C$. Thus, $\overline{\varphi\left(x_{i}\right) \varphi\left(x_{j}\right)}$ is disjoint from $P$ if and only if $A_{i}$ intersects $A_{j}$, so $\left\{\varphi\left(x_{i}\right)\right\}$ with $P$ is an obstacle representation of $G$.

The remaining lemmas show how to find a bounded $k$-points representation for any $k$-clique-coverable non-double-covering circular arc graph. The first step is the following theorem of Hell and Huang.

Theorem 12 ([14]) Suppose that a circular arc graph $G$ can be covered by $k \geq 2$ cliques. Then any circular arc representation of $G$ has a $k$-point transversal.

It remains to show that if $G$ has a non-double-covering circular arc representation with a $k$-point transversal, then $G$ has a bounded $k$-points representation.

Lemma 13 If $G$ has a non-double-covering circular arc representation with a $k$ point transversal, $k \geq 2$, then $G$ has a non-double-covering circular arc representation with a $k$-point transversal for which no arc of the representation contains all $k$ points.

Proof Consider a non-double-covering circular arc representation $R$ of $G$ with $k$ point transversal $T$ that minimizes the sum over all arcs $A \in R$ of the number of transversal points contained in $A$. We will show that no arc of $R$ contains all $k$ points of $T$.

For any points $p$ and $q$ of the circle, let $[p, q]$ denote the clockwise arc from $p$ to $q$. Suppose there is some arc $A=\left[a_{1}, a_{2}\right]$ containing every point of $T$. Let $t_{1}$ be the point of $T$ immediately clockwise from $a_{1}$, and $t_{2}$ the point of $T$ immediately counterclockwise from $a_{2}$. We claim that we can shorten $A$ by moving $a_{1}$ past $t_{1}$ and still have a representation of $G$. Then $A$ contains fewer points of $T$, contradicting minimality.

To show this, suppose there is some arc $B=\left[b_{1}, b_{2}\right]$ with $b_{2} \in\left[a_{1}, t_{1}\right]$, as in Fig. 15. Then because $B$ must contain some transversal point, $B$ must contain $t_{2}$ and therefore $a_{2}$, contradicting the assumption that $G$ is non-double-covering. Therefore no such $B$ exists, and moving $a_{1}$ past $t_{1}$ maintains all the intersections of $A$ with the other arcs.

An alternate proof of Lemma 13 for the case $k=2$ is given in [21]. The following lemma concludes the proof of Theorem 10 for the case $k \geq 3$. 
Lemma 14 If there exists a non-double-covering circular arc representation $R$ of graph $G$ with a transversal $T$ of size $k \geq 3$, in which no arc contains every point of $T$, then there exists a bounded k-points representation of $G$.

Proof Let $t_{1}, t_{2}, \ldots, t_{k}$ be the points of $T$, labeled clockwise around the circle. We create a collection $S$ of $k$ arcs $A_{1}, A_{2}, \ldots, A_{k}$ as follows. For $1 \leq i \leq k$, we place $A_{i}$ so that it contains the clockwise arc $\left[t_{i}, t_{i+1}\right]$ (where $t_{k+1}=t_{1}$ ) but no arc endpoints outside $\left[t_{i}, t_{i+1}\right]$. Then $R \cup S$ is non-double-covering, because for any $A_{i} \in S, B \in$ $R \cup S$ with $A_{i} \cup B$ equal to the whole circle, $B$ would contain all of $T$, contradicting the hypothesis of the lemma.

We apply Lemma 7 to $R \cup S$ to find a bounded representation $R^{*} \cup S^{*}$ with arc endpoints in the same order as in $R \cup S$, and find a transversal $T^{*}$ of size $k$ so that the arc endpoints of $R^{*}$ and $S^{*}$ and the points of $T^{*}$ all appear in the same order as in $R \cup S \cup T$. Suppose $H$ is a semicircle containing $T^{*}$. Then the complementary semicircle $H^{\prime}$ is between two consecutive points of $T^{*}$ and so must be contained in some arc of $S^{*}$. But every arc of $S^{*}$ is shorter than a semicircle, so no such $H$ exists. Thus, $R^{*}$ with $T^{*}$ is a bounded $k$-points representation of $G$.

For the case $k=2$, we define a bounded 2-points representation of a graph $G$ to be a bounded circular arc representation of $G$ together with a transversal $T$ consisting of two antipodal points of the circle. We extend the proof of Lemma 11 to the case $k=2$ by choosing the obstacle $P$ to be a segment oriented orthogonal to the line through the two transversal points. The remainder of the proof of the lemma is the same.

As with $k \geq 3$, a non-double-covering circular arc graph $G$ coverable by two cliques has a non-double-covering circular arc representation with a two-point transversal not fully contained by any arc, by Theorem 12 and Lemma 13. The following lemma concludes the proof of Theorem 10 .

Lemma 15 If there exists a non-double-covering circular arc representation $R$ of graph $G$ with a transversal $T$ containing exactly two points, in which no arc contains both points of $T$, then there exists a bounded 2-points representation of $G$.

Proof First, we use Lemma 7 to find a bounded representation $R^{*}$ of $G$ on a circle $C$ of circumference 1 with endpoints in the same order as in $R$, with transversal points $p$ and $q$ so that each arc in $R^{*}$ contains exactly one of $p$ and $q$. We replace $p$ by an arc $P$ of length 2, and replace $q$ by an arc $Q$ of length 2. $C$ is now a closed curve of total length 5. Each arc which contained $p$ now contains all of $P$, and each arc which contained $q$ now contains all of $Q$, so the length of every arc in $R^{*}$ increases by exactly 2 .

Preserving all lengths, make $C$ into a circle of total circumference 5. Any arc that had length $l<1 / 2$ now has length $(2+l)<5 / 2$, so the new representation is bounded. Some point $q^{*} \in Q$ is antipodal to some point $p^{*} \in P$, because the set of points antipodal to points in $P$ has length 2, while the set of points not in $P$ or $Q$ has length only 1 . We choose $\left\{p^{*}, q^{*}\right\}$ as our new transversal.

The proof of Theorem 10 is now complete. 
For the case $k=2$, a graph $G$ is a non-double-covering circular arc graph with clique covering number 2 if and only if $G^{C}$ is an interval bigraph [15]. In Sect. 7, we offer an alternate proof by proving directly that $G$ has a 2-gon representation if and only if $G^{C}$ is an interval bigraph.

\section{Segment Obstacle Representations and Interval Bigraphs}

The segment obstacle number of a graph $G$ is the least number of obstacles in any obstacle representation of $G$ in which all the obstacles are line segments.

An interval bigraph representation of a graph $G$ is a set of closed intervals on the real line, colored red and blue, in one-to-one correspondence with the vertices of $G$, such that two vertices of $G$ are adjacent if and only if their corresponding intervals intersect and are different colors. We frequently color the vertices $G$ with the colors of their corresponding intervals. If $G$ has an interval bigraph representation, then $G$ is an interval bigraph [4-6]. Note that all interval bigraphs are bipartite, because no two vertices of the same color are adjacent.

In [15], Hell and Huang show that $G^{C}$ is an interval bigraph if and only if $G$ is a non-double-covering circular arc graph with clique covering number 2 . Now suppose $G$ is a graph with segment obstacle number 1 . By Theorem 10 , we know $G$ is a nondouble-covering circular arc graph with clique covering number 2 . In Theorem 16, we present a construction that takes an interval bigraph representation of $G^{C}$ and produces a segment obstacle representation of $G$, and vice versa.

Theorem 16 A graph $G$ has segment obstacle number 1 if and only if $G^{C}$ is an interval bigraph.

Proof For a given interval bigraph representation $T$ of $G$, we assume without loss of generality that $T$ is a set of closed intervals on the line $y=1$ in the plane, and denote an interval $[(a, 1),(b, 1)], a<b$, by $[a, b]$. Similarly, for a given segment obstacle representation of $G$ with one segment $S$, we assume that $S$ is the line segment $[(-1,0),(1,0)]$ on the $x$-axis. Because we take vertices of $G$ and vertices of $S$ to be in general position, vertices in $T$ cannot be on the $x$-axis. We color vertices above the $x$-axis red and vertices below the $x$-axis blue.

We construct a bijection $\varphi$ from the set of closed intervals on $y=1$ to the set of points above the $x$-axis, such that two intervals $I$ and $J$ intersect if and only if the segment between $\varphi(I)$ and $-\varphi(J)$ (or equivalently, between $-\varphi(I)$ and $\varphi(J)$ ) intersects the segment $[(-1,0),(1,0)]$. Then given an interval bigraph representation $T$ on line $y=1$ of $G^{C}$, the set of points $\{\varphi(R) \mid R \in T$ is red $\} \cup\{-\varphi(B) \mid B \in T$ is blue $\}$ with the segment $[(-1,0),(1,0)]$ is a segment obstacle representation of $G$. Conversely, given a segment obstacle representation of $G$ with obstacle $[(-1,0),(1,0)]$, the set of intervals $\left\{\varphi^{-1}(r) \mid r \in V(G)\right.$ is red $\} \cup\left\{\varphi^{-1}(-b) \mid b \in V(G)\right.$ is blue $\}$ is an interval bigraph representation of $G^{C}$.

The construction is as follows. We define $\varphi([a, b])$ to be the intersection of two lines: one line through $(-1,0)$ parallel to the line through $(b, 1)$ and $(0,0)$, and the other through $(1,0)$ parallel to the line through $(a, 1)$ and $(0,0)$, as shown in Fig. 16. 
Fig. 16 The bijection $\varphi$ between an interval $[a, b]$ and a point $(x, y)$

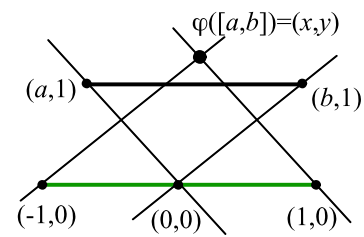

Note that $\varphi$ is invertible, because there is a corresponding geometric interpretation for the inverse function $\varphi^{-1}$.

Algebraically,

$$
\varphi([a, b])=\left(\frac{a+b}{b-a}, \frac{2}{b-a}\right)
$$

and

$$
\varphi^{-1}(x, y)=\left[\frac{x-1}{y}, \frac{x+1}{y}\right] .
$$

We will show that point $\varphi(J)$ is visible from $-\varphi(I)$ if and only if intervals $I$ and $J$ are disjoint. Observe that $\varphi(J)$ is to the right of the line through $-\varphi(I)$ and $(1,0)$ if and only if the left endpoint of $J$ is to the right of the right endpoint of $I$; that is, $-\varphi(I)$ sees $\varphi(J)$ to the right of $[(-1,0),(1,0)]$ if and only if $J$ is completely to the right of $I$. Likewise, $\varphi(J)$ is to the left of the line through $-\varphi(I)$ and $(-1,0)$ if and only if the right endpoint of $J$ is to the left of the left endpoint of $I$, so $-\varphi(I)$ and $\varphi(J)$ are mutually visible if and only if $I$ and $J$ are disjoint.

Thus we have constructed the desired bijection, and so $G$ has segment obstacle number 1 if and only if $G^{C}$ is an interval bigraph.

An interval k-graph representation of a graph $G$ is a set of closed intervals on the real line, colored with $k$ colors, in one-to-one correspondence with the vertices of $G$, such that two vertices of $G$ are adjacent if and only if their corresponding intervals intersect and are different colors. We frequently color the vertices of $G$ with the colors of their corresponding intervals. If $G$ has an interval $k$-graph representation, then $G$ is an interval $k$-graph [7]. Since $G$ is a non-double-covering circular arc graph with clique covering number 2 if and only if $G^{C}$ is an interval bigraph [15], it is natural to try replacing 2 by $k$ and hope that a graph $G$ is a non-double-covering circular arc graph with clique covering number $k$ if and only if $G^{C}$ is an interval $k$-graph. Unfortunately, neither implication is true. We provide two separating examples to show that, for $k>2$, neither of the classes of complements of interval $k$-graphs and non-double-covering circular arc graphs (equivalently, graphs with convex obstacle number 1) is contained in the other.

First, we show that not every interval $k$-graph is the complement of a non-doublecovering circular arc graph. Let $G$ be the disjoint union of $K_{2}$ and $K_{3}$. It is easy to check that $G$ is an interval 3-graph. However, $G^{C}$ is the complete bipartite graph $K_{2,3}$, which is not a non-double-covering circular arc graph (equivalently, not representable with a single convex obstacle) by Corollary 9 .

Now we show that not every complement of a non-double-covering circular arc graph is an interval $k$-graph. Consider the graph $C_{5}$, a non-double-covering circular 
arc graph. Brown, Flink, and Lundgren showed that if $G$ is an interval $k$-graph, then $G$ is weakly chordal; that is, neither $G$ nor $G^{C}$ contains a cycle of length at least 5 as an induced subgraph [3,7]. The graph $\left(C_{5}\right)^{C}=C_{5}$ is clearly not weakly chordal and thus is not an interval $k$-graph. Thus, $C_{5}$ is a non-double-covering circular arc graph, but its complement is not an interval $k$-graph for any $k$.

Acknowledgements We thank Arden Brookstein, Richard Marcus, and Andrew Ravits for defining obstacle numbers of graphs and posing questions answered in this paper, and we thank the anonymous referee. This research took place in the Willamette Valley REU-RET Consortium for Mathematics Research, sponsored by the National Science Foundation (grant number DMS 0649068) and Willamette University.

\section{References}

1. Bárány, I., Károlyi, G.: Problems and results around the Erdős-Szekeres convex polygon theorem. In: Discrete and Computational Geometry, Tokyo, 2000. Lecture Notes in Comput. Sci., vol. 2098, pp. 91-105. Springer, Berlin (2001)

2. Brass, P., Moser, W., Pach, J.: Research Problems in Discrete Geometry. Springer, New York (2005)

3. Brown, D.E.: Variations on interval graphs. PhD thesis, University of Colorado Denver (2004)

4. Brown, D.E., Lundgren, J.R.: Relationships among classes of interval bigraphs (0, 1)-matrices and circular arc graphs. In: Proceedings of the Thirty-Fifth Southeastern International Conference on Combinatorics, Graph Theory and Computing, vol. 166, pp. 97-123 (2004)

5. Brown, D.E., Lundgren, J.R., Miller, C.: Variations on interval graphs. In: Proceedings of the ThirtySecond Southeastern International Conference on Combinatorics, Graph Theory and Computing (Baton Rouge, LA, 2001), vol. 149, pp. 77-95 (2001)

6. Brown, D.E., Flink, S.C., Lundgren, J.R.: Characterizations of interval bigraphs and unit interval bigraphs. In: Proceedings of the Thirty-Third Southeastern International Conference on Combinatorics, Graph Theory and Computing, Boca Raton, FL, 2002, vol. 157, pp. 79-93 (2002)

7. Brown, D.E., Flink, S.C., Lundgren, J.R.: Interval $k$-graphs. In: Proceedings of the Thirty-Third Southeastern International Conference on Combinatorics, Graph Theory and Computing, Boca Raton, FL, 2002, vol. 156, pp. 5-16 (2002)

8. Deng, X., Hell, P., Huang, J.: Linear-time representation algorithms for proper circular-arc graphs and proper interval graphs. SIAM J. Comput. 25(2), 390-403 (1996)

9. Durán, G., Gravano, A., McConnell, R.M., Spinrad, J., Tucker, A.: Polynomial time recognition of unit circular-arc graphs. J. Algorithms 58(1), 67-78 (2006)

10. ElGindy, H.A.: Hierarchical decomposition of polygons with applications. PhD thesis, McGill University (1985)

11. Erdős, P., Szekeres, G.: A combinatorial problem in geometry. Compos. Math. 2, 463-470 (1935)

12. Fischer, P., Simon, H.-U.: Separation problems and circular arc systems. In: Graph-Theoretic Concepts in Computer Science, Berlin, 1990. Lecture Notes in Comput. Sci., vol. 484, pp. 251-259. Springer, Berlin (1991)

13. Golumbic, M.C.: Algorithmic Graph Theory and Perfect Graphs, 2nd edn. Annals of Discrete Mathematics, vol. 57. Elsevier, Amsterdam (2004). With a foreword by Claude Berge

14. Hell, P., Huang, J.: Two remarks on circular arc graphs. Graphs Comb. 13(1), 65-72 (1997)

15. Hell, P., Huang, J.: Interval bigraphs and circular arc graphs. J. Graph Theory 46(4), 313-327 (2004)

16. McConnell, R.M.: Linear-time recognition of circular-arc graphs. Algorithmica 37(2), 93-147 (2003)

17. McKee, T.A., McMorris, F.R.: Topics in Intersection Graph Theory. SIAM Monographs on Discrete Mathematics and Applications. Society for Industrial and Applied Mathematics (SIAM), Philadelphia (1999)

18. Morris, W., Soltan, V.: The Erdős-Szekeres problem on points in convex position-a survey. Bull. Am. Math. Soc. (N.S.) 37(4), 437-458 (2000) (electronic)

19. O'Rourke, J.: Art Gallery Theorems and Algorithms. International Series of Monographs on Computer Science. The Clarendon Press Oxford University Press, New York (1987)

20. Radziszowski, S.P.: Small Ramsey numbers. Electron. J. Comb., Dyn. Surv. 1, 60 pp. (1994-2006) (electronic) 
21. Spinrad, J.: Circular-arc graphs with clique cover number two. J. Comb. Theory Ser. B 44(3), 300-306 (1988)

22. Tóth, G., Valtr, P.: The Erdôs-Szekeres theorem: upper bounds and related results. In: Combinatorial and Computational Geometry. Math. Sci. Res. Inst. Publ., vol. 52, pp. 557-568. Cambridge Univ. Press, Cambridge (2005)

23. Tucker, A.: Characterizing circular-arc graphs. Bull. Am. Math. Soc. 76, 1257-1260 (1970)

24. Tucker, A.: Matrix characterizations of circular-arc graphs. Pac. J. Math. 39, 535-545 (1971)

25. West, D.B.: Introduction to Graph Theory, 2nd edn. Prentice Hall Inc., Upper Saddle River (2001) 